Copyright @ 2006 IEEE.

Reprinted from International Conference on Photonics in Switching (2006 : Heraklion, Crete)

:pp.158-160

This material is posted here with permission of the IEEE. Such permission of the IEEE does not in any way imply IEEE endorsement of any of the University of Adelaide's products or services. Internal or personal use of this material is permitted. However, permission to reprint/republish this material for advertising or promotional purposes or for creating new collective works for resale or redistribution must be obtained from the IEEE by writing to pubs-permissions@ieee.org.

By choosing to view this document, you agree to all provisions of the copyright laws protecting it. 


\title{
Chalcogenide Glasses for All-optical Processing
}

\author{
B. Luther-Davies ${ }^{1}$, S. J. Madden ${ }^{1}$, D-Y Choi ${ }^{1}$, R-P Wang ${ }^{1}$, A. V. Rode ${ }^{1}$, A. Prasad ${ }^{1}$, R. A. \\ Jarvis $^{1}$, D. Moss ${ }^{2}$, B. Eggleton ${ }^{2}$, C. Grillet ${ }^{2}$, M. R. E. Lamont ${ }^{2}$, E. Magi ${ }^{2}$, V. Ta'eed ${ }^{2}$, M. \\ Shookooh-Saremi ${ }^{2}$, N. Baker ${ }^{2}$, I. Littler ${ }^{2}$, L. Fu' ${ }^{2}$, M. Rochette ${ }^{2}$, Y. Ruan ${ }^{3}$, \\ Centre for Ultrahigh Bandwidth Devices for Optical Systems \\ 1: Laser Physics Centre, Australian National University, Canberra, ACT 0200 Australia \\ 2: School of Physics, University of Sydney, Sydney NSW 2006, Australia \\ 3: Centre of Expertise in Photonics, School of Chemistry and Physics, University of Adelaide, SA5005 Australia
}

\begin{abstract}
Chalcogenide glasses, which contain S, Se or Te atoms combined with network forming elements such as $\mathrm{Ge}, \mathrm{As}, \mathrm{Sb}$ have the largest third order optical nonlinearity of any inorganic glass. As a result they are attractive candidates for fibre and waveguide devices for all-optical signal processing in the telecommunications bands. In this talk I will review our recent progress in alloptical devices such as regenerators, wavelength converters and other devices in chalcogenide glasses.
\end{abstract}

Keywords: All-optical processing; chalcogenide glasses, devices

\section{Introduction}

Chalcogenide glasses containing the chalcogens $\mathrm{S}$, Se and Te combined with network forming elements such as As, Ge, Si are an important group of high index (n $\approx 2-3)$ infra-red transmitting glasses. According to Miller's rule [1], high linear index implies large ultra-fast optical nonlinearity making these glasses interesting for all-optical signal processing in high bit rate communications systems. Particularly important is the fact that these glasses have a combination of high refractive nonlinearity $\left(\mathrm{n}_{2} \approx 1000 \mathrm{x}\right.$ silica) and low absorptive nonlinearity $(\beta)$ which leads to favourable nonlinear figure of merit (FOM), $T=n_{2} / \beta \lambda>>1$. Thus these glasses should be suitable for fabricating alloptical switching devices operating above $100 \mathrm{~Gb} / \mathrm{s}$.

As well as their favourable nonlinear properties, chalcogenides are attractive for other reasons. They form glasses for a wide range of compositions and thus their refractive index to be tuned sensitively to obtain arbitrary core-cladding index contrast. Their high index allows the fabrication of single mode waveguides with sub-micron dimensions. This leads to strong field confinement that enhances the nonlinear response of devices. Additionally they have relatively high Verdet constants making magneto-optic structures feasible; they have shown potential to be poled leading to moderate second order nonlinearity [2]; they are photosensitive allowing inscription of gratings as well as direct writing of waveguides; and they display large Raman gain; etc.

The first demonstration of the use of chalcogenides for alloptical switching was in fact made by in the early 1990s by Asboe et al.[3]. Switching of a $40 \mathrm{GHz}$ pulse train using pulse powers as low as $0.4 \mathrm{~W}$ was reported using an $\mathrm{As}_{2} \mathrm{~S}_{3}$ glass fiber $4 \mathrm{~m}$ long.
Whilst this early result was encouraging many challenges remain before chalcogenides can be accepted for alloptical processing. Furthermore whilst most demonstrations so far have been performed in fiber geometry, the ultimate goal will be to demonstrate fast low power switching in integrated devices based on chalcogenide glass films. In this talk I summarise some of our recent results in all-optical processing using chalcogenides and outline our approach to low power switching in integrated devices.

\subsection{Fiber based all-optical regenerator}

To demonstrate the efficacy of chalcogenide glasses for all-optical processing we have recently reported [4] an alloptical regenerator based on the use single mode $\mathrm{As}_{2} \mathrm{Se}_{3}$ fiber. $\mathrm{As}_{2} \mathrm{Se}_{3}$ has significantly higher optical nonlinearity $\left(\approx 400 \mathrm{x}\right.$ silica) compared with the $\mathrm{As}_{2} \mathrm{~S}_{3}$ glass ( $\approx 100 \mathrm{x}$ silica) used by Asobe et al.[3] although its nonlinear absorption is higher yielding a nonlinear figure of merit, $T \approx 2.3$. However as will be shown below, it appears that this is an almost optimal value of the FOM for this particular application and leads to an near ideal optical transfer function for the regenerator.

The regenerator is based on the design suggested by Mamyshev in 1998 (figure 1) [5]. A noisy return to zero input signal is passed through a dispersive and nonlinear medium $\left(\mathrm{As}_{2} \mathrm{Se}_{3}\right.$ fiber with $D=-504 \mathrm{ps} / \mathrm{nm} / \mathrm{km} ; n_{2}=0.910^{-}$ ${ }^{13} \mathrm{~cm}^{2} / \mathrm{W}$; and $\beta=2.510^{-12} \mathrm{~m} / \mathrm{W}$ ) producing self phase modulation (SPM) induced spectral broadening. Low power noise experiences minimal SPM spectral broadening and so is filtered out the band-pass filter offset from the input centre wavelength. On the other hand high power pulses experience sufficient spectral broadening to be partially transmitted through the bandpass filter. This results in a step-like power transfer function that suppresses noise in both the "zeros" and "ones" in the data stream.

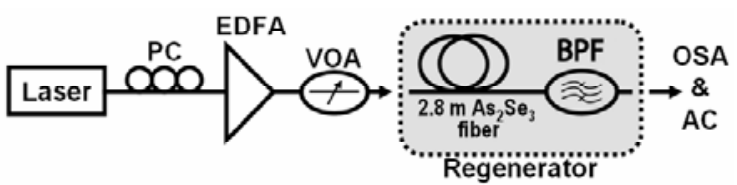

Figure 1: Experimental configuration for demonstrating optical regeneration. PC - polarization controller, EDFA erbium doped fiber amplifier, VOA - variable optical 
attenuator, BPF - bandpass filter, OSA - optical spectrum analyzer and $A C$ - pulse autocorrelator.

The measured transfer function for our device is shown in figure 2 along with model predictions including dispersion and illustrating the effect of the finite level of two photon absorption. Clearly an almost ideal step like transfer function can be obtained using this glass at least using fibers a few meters long. Frequency Resolved Optical Gating (FROG) confirmed that the output pulses were near transform limited with pulse durations close to those of the incoming pulse train.

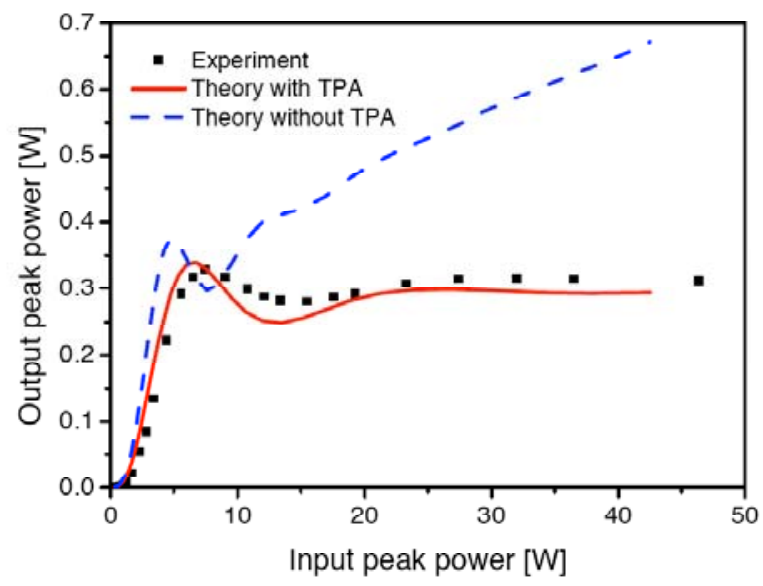

Figure 2: Regenerator transfer function for a filter offset of $1.35 \mathrm{~nm}$. Experiment compared to theory, with and without two photon absorption.

\subsection{Towards an Integrated Regenerator}

Whilst this demonstration using fiber indicated the feasibility of using the chalcogenides for all-optical processing, integrated devices are far preferable and would allow fabrication of compact devices for regenerating multiple WDM channels on a single wafer. To this end we are developing methods for processing low loss chalcogenide glass films to create compact high index contrast planar waveguides for all-optical processing.

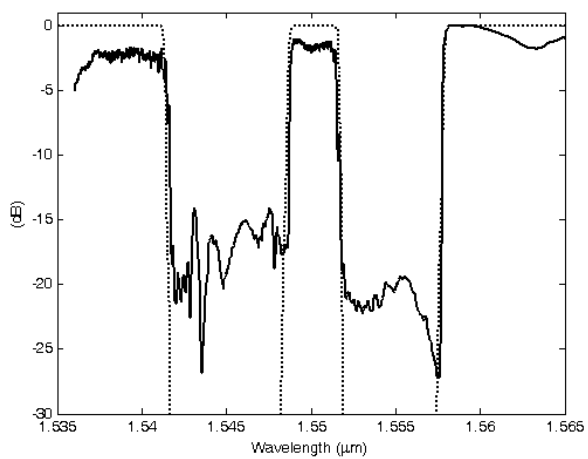

Figure 3: Experimental (solid) and theoretical (dotted) transmission spectra for the pass-band filter, consisting of two Bragg gratings. The longer wavelength grating was written closest to the output facet of the waveguide. Pass and rejection bands are respectively $2.8 \mathrm{~nm}$ and $16.3 \mathrm{~nm}$ wide

We have shown that a combination of ultra-fast pulsed laser deposition combined with conventional dry etching can be used to produce relatively low loss $(\approx 0.2 \mathrm{~dB} / \mathrm{cm})$ chalcogenide glass rib waveguides [6]. Furthermore the inherent photosensitivity of chalcogenides to near-bandgap light has allowed us to write strong Bragg gratings required for the regenerators directly into these waveguides [7]. A sample filter response used in a prototype waveguide all-optical regenerator is shown in figure 3 .

In a waveguide device $5 \mathrm{~cm}$ long we have shown a nonlinear transfer function could be obtained [8] although the operating power of this prototype was around 50W well above the level required for telecommunications applications. Considerable potential exists however to reduce the required power by increasing the device length (from $5-50 \mathrm{~cm}$ ); reduce the mode area (by around a factor of 5) and increase the nonlinearity by choosing a different glass (by a factor of 4-5). Thus operating powers of $<1 \mathrm{~W}$ appear possible.

\subsection{Wavelength conversion in a chalcogenide rib waveguide}

A Mamyshev regenerator inevitably causes a wavelength shift at the output relative to the input and hence integrated devices should be capable of wavelength conversion to restore the original signal wavelength (or alternatively shift the output to a new WDM channel). We have recently demonstrated all-optical wavelength conversion in an $\mathrm{As}_{2} \mathrm{~S}_{3}$ rib waveguide using cross phase modulation. The principle of operation is shown in figure 4 .

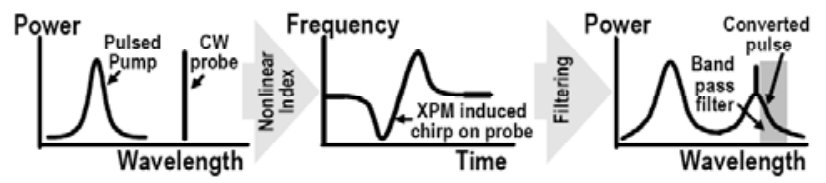

Figure 4 Principle of operation of wavelength conversion via cross-phase modulation (XPM) followed by filtering.

A pulsed pump source, potentially containing digital data, is directed though the nonlinear medium along with a CW probe beam near the desired output wavelength. The pump beam induces a transient chirp on the probe beam via cross phase modulation through the Kerr nonlinearity. This broadens the probe spectra generating sidebands, and when a single sideband is selected using an optical filter, the output signal is modulated in time similarly to the pump pulse.

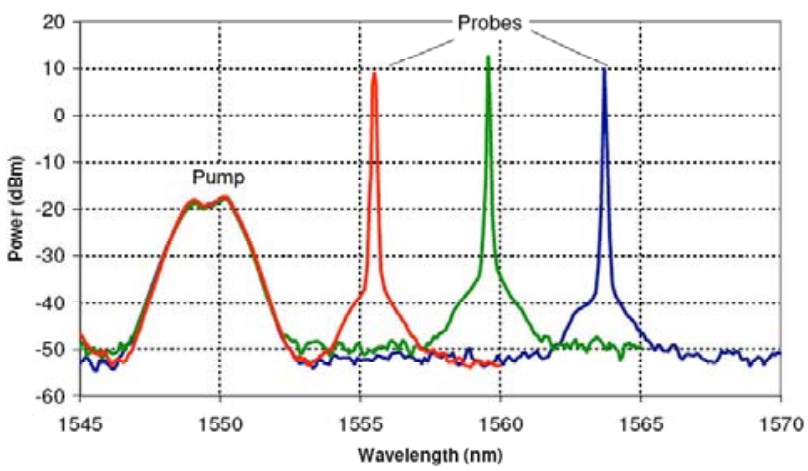

Figure 5 Unfiltered output spectra showing $2 \mathrm{~nm}$ wide pump spectra (left) and CW probe spectra (right) with optical sidebands imposed by XPM. 
Figure 5 shows the spectra of the unfiltered output signal containing both the pump ( $2 \mathrm{~nm}$ wide signal at $1550 \mathrm{~nm})$ and $\mathrm{CW}$ probes set to three different wavelengths $(1555 \mathrm{~nm}, 1560 \mathrm{~nm}$ and $1565 \mathrm{~nm})$. The sidebands on each probe are clearly visible. Note that the strength of the sidebands are low in this demonstration because of the low duty cycle (5ps pulses at $9 \mathrm{MHz}$ ) used in these experiments. For a full $33 \% \mathrm{RZ}$ signal the relative strength of the sidebands would be increased by about $40 \mathrm{~dB}$. After filtering to isolate the upper sideband in figure 5 the pulses were analysed using FROG that showed that the phase and amplitude integrity of the pulse in both the time and frequency domains was maintained by the wavelength converter. Because of the short waveguide length, pump/probe walk-off as well as pulse dispersion were not a limiting factor in device performance.

\subsection{Towards lower operating power}

The waveguide based devices described above generally required powers in excess of those available in optical communications system. As indicated in section 2,2, however, a clear route exists to reducing the operating powers to sub-W levels by scaling the physical dimensions of the devices. However, further reduction, can only be achieved by a radical change in device design.

A common theme in research to produce low power photonic switching structures is to employ high-Q resonators to reduce the external switching power. $A$ popular route to achieving this involves the fabrication of compact optical resonators in photonic crystal lattices. Unlike low index glasses, chalcogenides are suitable for fabricating 2-D photonic crystal lattices because of their high index and hence this route can also be used to reduce operating power albeit at the expense of increased switching times.

We have demonstrated the fabrication of high quality 2-D photonic crystal lattices in $\mathrm{Ge}_{33} \mathrm{As}_{12} \mathrm{Se}_{55}$ glass films [9]. Defect mode waveguides and resonators in these lattices have been probed via evanescent coupling from a tapered optical fiber (figure 6) [10].

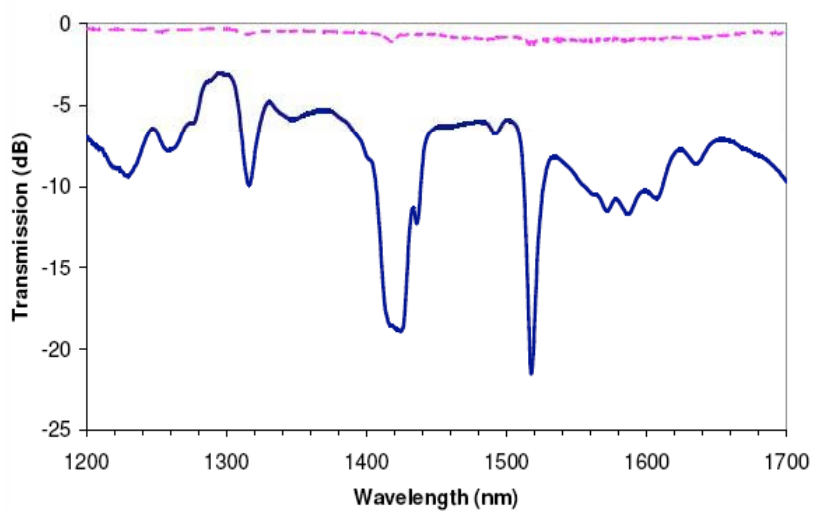

Figure 6: Transmission spectrum of tapered nanowire for TE polarized light, in close proximity to chalcogenide glass photonic crystal waveguide, showing resonant coupling. Dashed curve is for large $(>2 \mu \mathrm{m})$ fibre-waveguide separation. Solid curve is for close coupling ( $<1 \mu \mathrm{m}$ separation).

More than $98 \%$ coupling can be achieved in this geometry opening up the way for the demonstration of all-optical switching in micro-resonators based on photonic crystal structures in chalcogenides.

\section{Conclusion}

We have shown that chalcogenide glasses have considerable potential for all-optical signal processing. Their excellent nonlinear properties suggest that ultra-fast all-optical switching should be possible at pulse powers of a few hundred $\mathrm{mW}$ in chalcogenide nanowire waveguides. Lower operating powers should be possible using resonators embedded in photonc crystal waveguides.

\section{Acknowledgment}

The authors gratefully acknowledge the support for this work by the Australian Research Council through it Centres of Excellence, Discovery and Federation Fellow programs.

\section{References}

[1] R. C. Miller, Appl. Phys. Lett., 'Optical second harmonic generation in piezoelectric crystals' 5, 17 (1964).

[2] M. Guinard, V. Nazabal, F. Smektala, H. Zeghlache, A. Kudlinski, Y. Quiquempois, G. Martinelli, 'High second order nonlinear susceptibility induced in chalcogenide glasses by thermal poling' Optics Express 14, 1524 (2006).

[3] Asobe, M.; Itoh, H.; Miyazawa, T.; Kanamori, T. 'Efficient and ultrafast all-optical switching using high Dn, small core chalcogenide glass fiber.' Electronics Letters 29(22), 1966-8 (1993). Asobe, M.; Ohara, T.; Yokohama, I.; Kaino, T., ' Low power all-optical switching in a nonlinear optical loop mirror using chalcogenide glass fiber'. Electronics Letters (1996), 32(15), 1396-1397.

[4] L. B. Fu, M. Rochette, V. G. Ta'eed, D. J. Moss and B. J. Eggleton 'Investigation of self-phase modulation based optical regeneration in single mode $\mathrm{As}_{2} \mathrm{Se}_{3}$ chalcogenide glass fiber', Optics Express 13, 7639 (2005).

[5] P. V. Mamyshev, "All-optical data regeneration based on self-phase modulation effect", in Proc. European Conference on Optical Communications (ECOC'98), p 475, 1998.

[6] Y. Ruan, W. Li, Weitang; R. Jarvis; N. Madsen A. Rode, Andrei; B. Luther-Davies,. 'Fabrication and characterization of low loss rib chalcogenide waveguides made by dry etching'. Optics Express (2004), 12(21), 5140-5145.

[3] Shokooh-Saremi, Mehrdad; Ta'eed, Vahid G.; Baker, Neil J.; Littler, Ian C. M.; Moss, David J.; Eggleton, Benjamin J.; Ruan, Yinlan; Luther-Davies, Barry. 'High-performance Bragg gratings in chalcogenide rib waveguides written with a modified Sagnac interferometer.' Journal of the Optical Society of America B: Optical Physics (2006), 23(7), 1323-1331.

[8] Ta'eed, Vahid G.; Shokooh-Saremi, Mehrdad; Fu, Libin; Littler, Ian C. M.; Moss, David J.; Rochette, Martin; Eggleton, Benjamin J.; Ruan, Yinlan; Luther-Davies, Barry. 'Self-phase modulation-based integrated optical regeneration in chalcogenide waveguides.' IEEE Journal of Selected Topics in Quantum Electronics (2006), 12(3), 360-370.

[9] Freeman, Darren; Madden, Steve; Luther-Davies, Barry. 'Fabrication of planar photonic crystals in a chalcogenide glass using a focused ion beam.' Optics Express (2005), 13(8), 3079-3086.

[10] C Grillet, C Smith, Eric Magi, D Freeman, S Madden, B Luther-Davies, D J. Moss, B J. Eggleton 'Efficient Coupling to Chalcogenide Glass Photonic Crystal Waveguides via Tapered Optical Fiber Nanowires', Proceedings of CLEO 2006 paper CTuK4. 